\title{
Stripping Voltammetry with Semi-Circular Potential Waves: Reversible Systems
}

\author{
Yuki Uchida, Enno Kätelhön, and Richard G. Compton \\ Department of Chemistry, Physical and Theoretical Chemistry Laboratory, Oxford University, South Parks \\ Road, Oxford, OX1 3QZ, United Kingdom
}

\begin{abstract}
We investigate stripping voltammetry using a semi-circular potential wave. Our results show that the herein introduced method greatly amplifies the current compared to the conventional linear-sweep method and can thus markedly increase the sensitivity of voltammetric stripping over linear-sweep methods. The shape of the obtained voltammograms features a characteristic dependency on the analyte formal potential which may be exploited to gain additional selectivity.
\end{abstract}

\section{Introduction}

Stripping voltammetry allows the detection of metals, metal complexes ${ }^{1}$ and organic substances at low concentrations ${ }^{2}$ making it a valuable technique for trace analysis. ${ }^{3}$ Methods can be categorized into three types: anodic (ASV), cathodic (CSV), and adsorptive (AdSV). In this paper we focus on CSV, which, computationally is equivalent to ASV. Both, CSV and ASV require a pre-concentration step where the analyte is electrodeposited on the electrode surface by applying the appropriate potential for a certain amount of time followed by the dissolution step. ${ }^{2 ; 4}$ On the other hand, in AdSV, the analyte adsorbs on the electrode surface via other physical or chemical interaction which may not involve a charge transfer with the electrode. ${ }^{4 ; 5}$ The analysis in all three methods is conventionally carried out by changing the applied potential linearly with respect to time to either a negative or positive value depending on the desired reaction: ${ }^{6-9}$

$$
E(t)= \pm \nu t+E(t=0)
$$

The current response recorded via stripping voltammetry can be used to obtain analytical information such as the identity of the analyte through the peak potential and its concentration through the peak current ${ }^{10}$ and the charge under the curve. ${ }^{4}$ Stripping voltammetry is generally regarded as a highly sensitive method ${ }^{11-13}$ with detection limits going as low as $10^{-10} M$ in some cases. ${ }^{2}$

We herein propose a new method for stripping voltammetry that enhances the sensitivity of detection through the application of a semi-circular potential wave rather than the linear potential wave (1). It is shown that the semi-circular potential wave increases the peak current and produces a characteristic voltammetric curve for a given potential window and formal potential, and its shape changes with the shift of the potential window. The increased current allows the determination of surface concentrations 
and the characteristic curve is useful in identifying species of interest. We note that the classical system would be one using a mercury film electrode, however, the herein introduced model can be extended to similar systems with a conducting film.

\section{Theory}

In the following sections, a theoretical model for stripping voltammetry with a semi-circular potential waveform is explained and dimensionless coordinates are introduced. Furthermore, we discuss the semicircular wave and give a discription of the simulation method employed.

\section{$2.1 \quad$ Theoretical model}

We investigate a one-electron electrochemically-reversible reduction of $A$ to $B$ where species $A$ is adsorbed to the electrode in a film of thickness $d$ and $B$ is in solution.

$$
A(a d s)+e \rightleftharpoons B(s o l)
$$

As the system is fully reversible, it establishes a Nernstian equilibrium at the electrode surface at all times: ${ }^{2}$

$$
\begin{aligned}
E & =E_{f}^{0}+\frac{R T}{F} \ln \frac{c_{A}^{0}}{c_{B}^{0}} \\
& =E_{f}^{0}+\frac{R T}{F} \ln \frac{c_{A}^{s}}{d c_{B}}
\end{aligned}
$$

where $E$ is the electrode potential, $E_{f}^{0}$ is the formal potential of the $A / B$ couple, $R, T$ and $F$ hold their usual meanings, and $c_{A}^{0}$ and $c_{B}^{0}$ are the volume concentrations of species $A$ and $B$ at the electrode surface respectively. Here, since $A$ is adsorbed to the electrode, its concentration is expressed in terms of a surface concentration $c_{A}^{s}\left(\mathrm{~mol} \mathrm{~cm}^{-2}\right)$, which, divided by the film thickness $d$, gives the volume concentration $c_{A}^{0}$ at the electrode surface. Consequently, only the mass transport of $B$ is considered which we model via Fick's second law in one dimension: ${ }^{14 ; 15}$

$$
\frac{\partial c_{B}}{\partial t}=D_{B} \frac{\partial^{2} c_{B}}{\partial x^{2}}
$$

where $D_{B}$ is the diffusion coefficient of $B$.

In order to simulate the given system, five equations must be considered. First is the Nernst equation as given in Equation (3), which dictates the reaction at the electrode/solution interface. Second is the diffusion of $B$ as shown through Equation (4). We additionally need to consider, third and fourth, the fluxes of species $A$ and $B$ and fifth the conservation of mass as expressed by Equation (5), (6), and (7), respectively.

$$
\begin{gathered}
j_{A}=\frac{\partial c_{A}^{s}}{\partial t} \\
j_{B}=-D_{B} \frac{\partial c_{B}}{\partial x}
\end{gathered}
$$




$$
j_{A}=-j_{B}
$$

$j_{A}$ is the reaction rate density of $A$ in moles per unit per surface area $\left[\mathrm{mol} \mathrm{s}^{-1} \mathrm{~m}^{-2}\right]$, the rate at which the species reacts per surface area. For $j_{B}$, the reaction rate density is equivalent to the flux density in a normal direction away from the electrode surface.

Boundary conditions must be established to solve the diffusion equation. Initially, only $A$ exists at the electrode surface in which the concentration is assumed to be uniform within the film, and the solution concentration of all species is uniformly zero across all space.

$$
\begin{array}{r}
t \leq 0, x=0: c_{A}=c_{A}^{s}, c_{B}=0 \\
t \leq 0, x>0: c_{A}=0, c_{B}=0
\end{array}
$$

As $t$ increases, $A$ is consumed and $B$ is released into the bulk solution but the concentrations of both species remain 0 at the outer boundary:

$$
t>0, x \rightarrow \infty: c_{A}=0, c_{B}=0
$$

\subsection{Dimensionless coordinates}

Table 1 introduces the dimensionless coordinate transformations used in the simulation akin to those reported in our previous works. ${ }^{14}$ For this model, however, we normalize the parameters with respect to the film thickness, $d$, rather than the electrode radius, $\epsilon$, and $c_{A}^{*}$ is the initial volume concentration of species $A$ on the electrode surface.

Table 1: Dimensionless parameters. ${ }^{14}$

\begin{tabular}{ll}
\hline Parameter & Normalization \\
\hline concentration A & $C_{A}=\frac{c_{A}^{s}}{d c_{A}^{*}}$ where $c_{A}^{*}=\frac{c_{A}^{s}}{d}$ at $t=0$ \\
concentration B & $C_{B}=\frac{c_{B}}{c_{A}^{*}}$ \\
diffusion coefficient & $d_{B}=\frac{D_{B}}{D_{B}}=1$ \\
spatial coordinate & $X=\frac{x}{d}$ \\
time & $\tau=\frac{D_{B} t}{d^{2}}$ \\
potential & $\theta=\left(\frac{F}{R T}\right)\left(E-E_{f, A / C}^{0}\right)$ \\
scan rate & $\sigma=\left(\frac{d^{2}}{D_{B}}\right)\left(\frac{F}{R T}\right) \nu$ \\
current & $J=\frac{d I}{\pi \epsilon^{2} F D_{B} c_{A}^{*}}$ \\
\hline
\end{tabular}

Given the above transformations, we can write equations (3), (4), (5), (6), and (7) in dimensionless coordinates as follow:

$$
e^{\theta}=\frac{C_{A}^{0}}{C_{B}^{0}}
$$




$$
\begin{gathered}
\frac{\partial C_{B}}{\partial \tau}=\frac{\partial^{2} C_{B}}{\partial X^{2}} \\
J_{A}=\frac{\partial C_{A}^{0}}{\partial \tau} \\
J_{B}=-\left.\frac{\partial C_{B}}{\partial X}\right|_{X=0} \\
J_{A}=-J_{B}
\end{gathered}
$$

where $C_{A}^{0}$ and $C_{B}^{0}$ are the dimensionless volume concentrations of $A$ and $B$ at the electrode surface respectively. An important difference to note between the dimensional and dimensionless expressions is the change in the concentration of $A$. The former is written in terms of a surface concentration whereas the latter is written in terms of a volume concentration. As a result, the film thickness becomes irrelevant to the simulation model.

\subsection{Semi-circular potential wave}

We apply the conventional linear wave and the semi-circular wave established in our previous work ${ }^{16}$ given as:

$$
\theta(\tau)= \pm \sqrt{\left|A^{2}-\left[\sigma_{\text {avg }} \cdot\left(\tau-c \tau_{\max }\right)\right]^{2}\right|}+\theta_{\text {shift }}
$$

where $\theta(\tau)$ is the dimensionless potential with respect to dimensionless time, $\tau$, and $A$ is the amplitude of the wave, $\sigma_{a v g}$ is the dimensionless average scan rate, $c$ is the fraction of the maximum time when the centre of each semi-circle is observed, $\tau_{\max }=\frac{2 A}{\sigma_{\text {avg }}}$, and $\theta_{\text {shift }}=\theta_{\text {avg }}-\theta_{f, A / B}^{0} \cdot \theta_{f, A / B}^{0}$ is the dimensionless formal potential of the $A / B$ couple which is zero in accordance with the definition of the dimensionless coordinate transformations. When running experiments, the key parameter to consider is the $\theta_{\text {shift }}$, which in dimensional terms is defined as $E_{\text {shift }}=E_{\text {avg }}-E_{f}^{0}$ where $E_{\text {avg }}$ is the average of the potential window defined as $E_{\text {avg }}=\frac{E_{\text {initial }}+E_{\text {final }}}{2}$. Thus $E_{\text {shift }}$ is the difference between the midpoint of the potential window and the formal potential of the redox couple, and $\theta_{\text {shift }}$ is its dimensionless form. In contrast to the conventional linear waveform where the effect of varied scan rates is of interest, in the semi-circular waveform method, we are concerned with the effect of the set potential window on the current response as explained in later sections.

Figure 1 shows the two potential waves used; the light blue line represents the linear potential wave and the green line represents the semi-circular potential wave, both centred at $\theta=0$, i.e. $\theta_{\text {shift }}=0$, and $A=20$. The linear potential wave features a constant scan rate throughout the entire sweep as evident from the figure, whereas the semi-circular potential wave exhibits different temporal scan rates with an instantaneously infinitely fast scan rate at the mid-point of the potential wave. It is this latter feature which offers the enhanced sensitivity. 


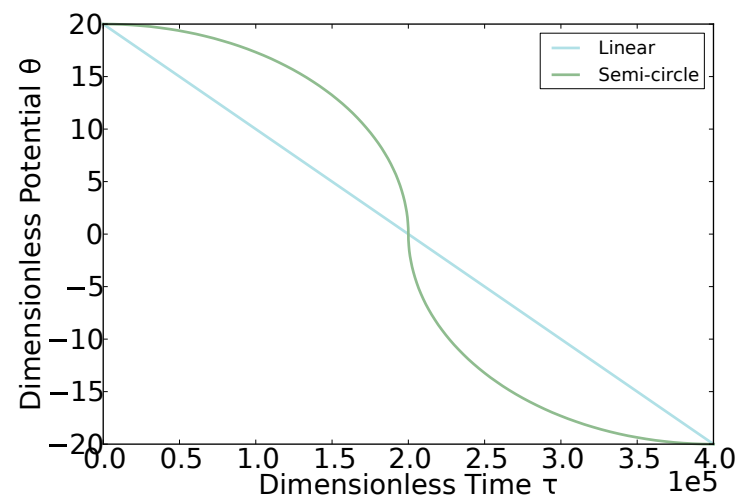

Figure 1: Linear and semi-circular potential wave graphs centered at 0 with an amplitude of 20 . The linear potential wave shows a constant scan rate throughout the duration of the sweep whereas the semi-circular potential wave shows the highest instantaneous scan rate at $\theta=0$ but rapidly decreases as the potential becomes further from 0 .

\subsection{Simulation via finite difference}

The simulation was performed by solving the discretized dimensionless diffusion equation via the finite difference method in $C++.{ }^{14}$ Plotting was done in Python using the packages NumPy and matplotlib. Numerous tests ${ }^{17}$ were conducted by comparing simulated results with theoretical expressions where available and through convergence tests.

\section{Results and discussion}

The following sections discuss the characteristic features arising from the application of a semi-circular potential wave in CSV and their advantage in detecting analytes at low concentrations. All results are reported for a dimensionless scan rate of $\sigma=10^{-4}$ unless otherwise specified. $\sigma=10^{-4}$ corresponds to approximately $\nu=257 \mathrm{mV} \mathrm{s}^{-1}$ assuming $D_{B}=10^{-9} \mathrm{~m}^{2} \mathrm{~s}^{-1}, d=10^{-7} \mathrm{~m}$, and $T=298 \mathrm{~K}$. Other parameters include $\Delta \theta=10^{-4}, \omega=1.07$, and $h=10^{-4}$ which are used in the finite differences method where $\Delta \theta$ is the difference in potential between each discritized time steps, $\omega$ is the expanding factor used for the expanding grid ${ }^{18}$, and $h$ is the initial difference between two points in the expanding spatial grid.

\subsection{Effect of potential window shift [Sections 3.1 and 3.2 have been swapped]}

Based on the potential waveform, we can expect characteristic voltammograms from the semi-circular method. [text deleted] The following section investigates how the voltammetric features depend on shifts of the potential window chosen for the semi-circular potential wave.

Figure 2 shows four voltammograms recorded at different $\theta_{\text {shift }}$ values (defined in Section 2.3) where Figure $2 A$ is for $\theta_{\text {shift }}=0$, Figure $2 B$ is for $\theta_{\text {shift }}=5$, Figure $2 C$ is for $\theta_{\text {shift }}=10$, and Figure $2 D$ is for $\theta_{\text {shift }}=15$. In all figures, the light line represents the result from the linear wave and the green line represents the result from the semi-circular wave. We see that Figure $2 A$ shows [text deleted] a broad peak and a sharp peak [text deleted]. The voltammetric shape when $\theta_{\text {shift }}=0$ is discussed in more detail in Section 3.2. At a more positive $\theta_{\text {shift }}$ of 5 we see one big peak such as in Figure $2 B$, and then at a more significantly positive $\theta_{\text {shift }}$, the sharp peak diminishes and only the broad peak is observable.

In $2 B$, the two peaks seen in $2 A$ merge and the current response is much larger than that obtained 


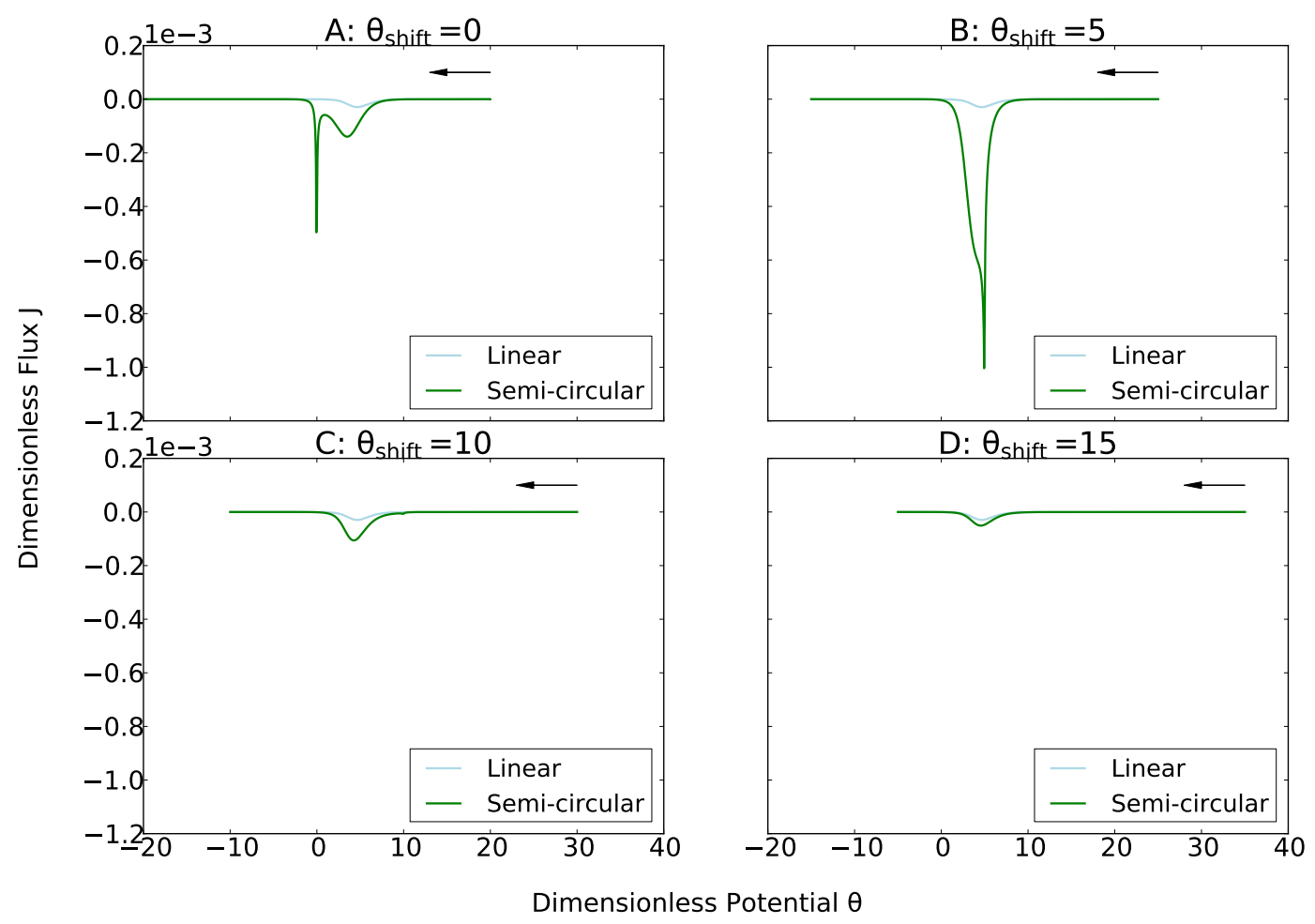

Figure 2: Voltammograms plotted for various $\theta_{\text {shift }}$ values. $A$ corresponds to $\theta_{\text {shift }}=0, B$ corresponds to $\theta_{\text {shift }}=5$, $C$ corresponds to $\theta_{\text {shift }}=10$, and $D$ corresponds to $\theta_{\text {shift }}=15$.

from the linear wave. This can be understood as, at $\theta=5$, the potential at which the infinitely fast scan rate occurs coincides with the potential region where the broad peak is otherwise observed. In $2 C$ and $2 D$ only one peak is observed as well which features a different shape than the peak in $2 B$. Here, the infinitely fast scan rate responsible for the sharp peak occurs at a highly positive potential where no reaction can proceed and thus does not affect the current response. In addition, the temporal scan rate at the potential region where the reaction takes place is relatively slow and produces a current response similar to that of the linear method.

The effect of the potential window shift is more apparent when the peak flux of each voltammogram is plotted against its corresponding $\theta_{\text {shift }}$ values as in Figure 3a. The solid light blue circular line represents the peak flux obtained from the linear sweep method, the solid light green triangular line represents the peak flux of the sharp peak from the semi-circular waveform method, and the dotted orange line represents the peak flux of the broad peaks. The peak flux from the linear method shows no variance with respect to $\theta_{\text {shift }}$, while the sharp peaks show a significant change in value depending on the shift. The broad peaks appear in the lower $\theta_{\text {shift }}$ and in the higher $\theta_{\text {shift }}$ region.

The linear method produces the same peak flux regardless of the potential shift since the wave provides a constant scan rate throughout the sweep and the scan window is chosen to be sufficiently large. In contrast, the semi-circular method exhibits an absolute maximum peak flux at a specific $\theta_{\text {shift }}$, and this value varies depending on the scan rate. The reason the broad peak currents are not plotted at certain $\theta_{\text {shift }}$ values is because in this potential range, the two peaks merge and have thus been plotted as a single sharp peak in the Figure. Figure $3 \mathrm{~b}$ also plots the dimensionless peak flux against the corresponding 


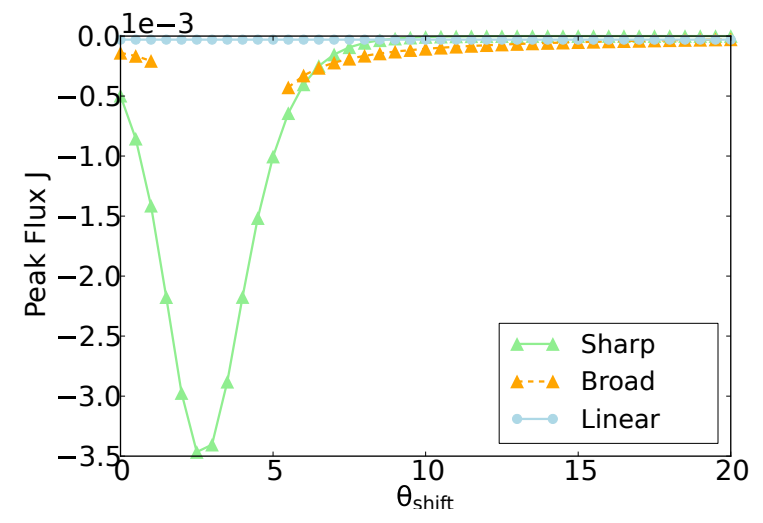

(a) Dimensionless peak flux plotted against corresponding $\theta_{\text {shift }}$ values at $\sigma=10^{-4}$.

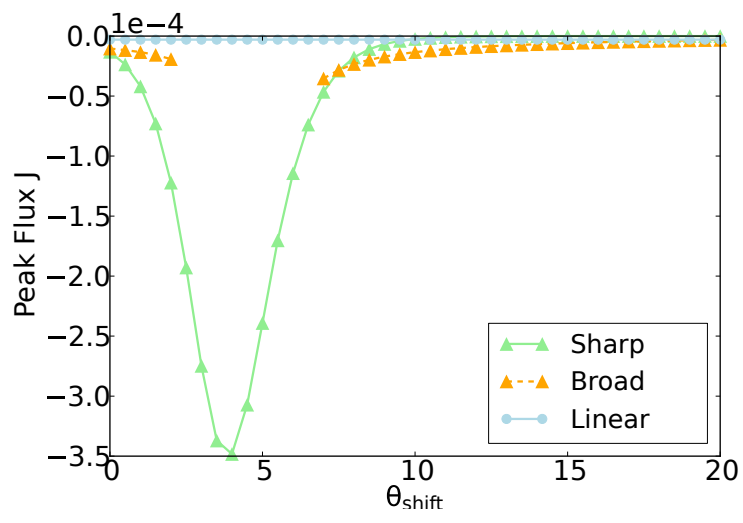

(b) Dimensionless peak flux plotted against corresponding $\theta_{\text {shift }}$ values at $\sigma=10^{-5}$.

Figure 3: Peak flux values plotted against varying $\theta_{\text {shift }}$ values at (a) $\sigma=10^{-4}$ and (b) $\sigma=10^{-5}$. The solid light blue circular line represents the peak flux from the linear method, the solid light green triangular line represents the peak flux of the sharp peak from the semi-circular method, and the dotted orange line represents the peak flux of the broad peak from said method.

$\theta_{\text {shift }}$ values but at a different scan rate of $\sigma=10^{-5}$. While the absolute maximum peak flux is observed at $\theta_{\text {shift }}=2.5$ in Figure $3 \mathrm{a}$, in Figure $3 \mathrm{~b}$ it is observed at $\theta_{\text {shift }}=4$ hence illustrating the scan rate dependency of the method.

\subsection{Features of semi-circular potential wave}

As evident from the previous section, the semi-circular method introduces attractive features to the voltammetric response. In this section we [text deleted] discuss the difference in voltammetric features between the linear and semi-circular potential waves when $\theta_{\text {shift }}=0$. Figure 4 shows the dimensionless current responses for linear and semi-circular potential waves at $\theta_{\text {shift }}=0$ where the light blue line represents the result from the linear wave and the green line represents the result from the semi-circular wave. The pink horizontal and vertical dotted lines are the theoretically predicted peak current and peak potential values plotted from Equation (16) ${ }^{11 ; 19-21}$ and (17) ${ }^{20 *}$ respectively:

$$
\begin{gathered}
I_{\text {peak }}=0.2975 n F A \sigma d c_{A}^{*} \\
n\left(E_{p}-E_{f}^{0}\right)=-1.43+12.85 \ln \left(\frac{d^{2} \sigma}{D_{B}}\right)[m V]
\end{gathered}
$$

where $n$ is the number of electrons involved in the reaction, $A$ is the area of the electrode, and $\sigma=\frac{n F}{R T} \nu$. We note that while the recorded flux of $B$ is a positive value, the plotted dimensionless current is negative as per convention to show a reduction is modelled.

From Figure 4, it is clear that the semi-circular potential wave gives a significantly larger current response compared to the linear method, which results in a higher sensitivity. As seen in Figure 1, semi-circular potential wave gives rise to different temporal scan rates throughout the sweep with an instantaneously infinitely fast scan rate at the mid-point of the potential window. The larger current response is due to the increase in temporal scan rate in the potential region where the electrochemical

\footnotetext{
(17).

${ }^{*}$ We note that the respective equation is misprinted in the original publication ${ }^{20}$ which we have corrected in Equation
} 


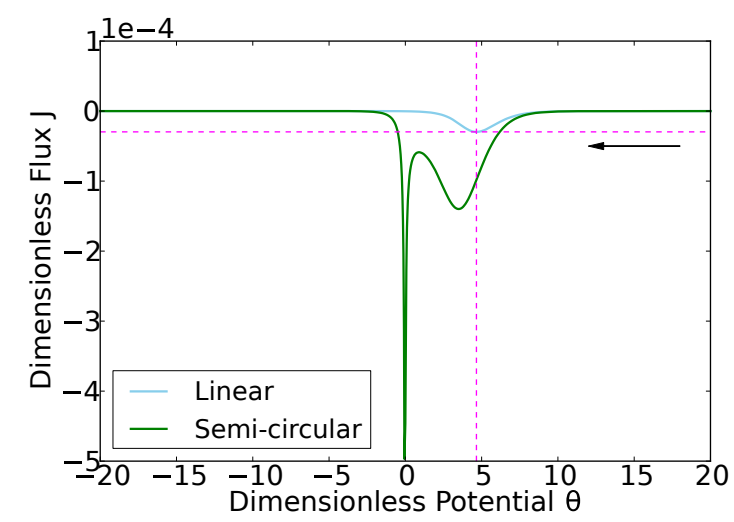

Figure 4: Dimensionless current response using linear and semi-circular potential waves at $\theta_{\text {shift }}=0$. The light blue line represents the result from the linear wave while the green line represents the result from the semi-circular wave. The pink horizontal and vertical dotted lines are the peak current and peak potential values predicted using Equations (16) and (17).

reaction occurs. The different temporal scan rates also explain the presence of a double peak. In Figure 4, we see a broad peak near the region where the peak is observed for the linear wave and, in addition, a sharp peak later in the sweep. There are two factors affecting the broad peak: one is the increasing scan rate and the other is the depletion of $A$. When the reaction starts near $\theta=10$, a large amount of $A$ is [text deleted] at the electrode which is consumed at an increasing rate along with an increasing scan rate, resulting in a higher current as in the case of a linear potential wave. In contrast to the linear wave voltammogram where the current falls off past $\theta=5$ due to depletion of the materials, the current in the semi-circular voltammogram continues to grow because of the effect of the increasing scan rate. Beyond $\theta=3$, the depletion dominates and the current falls until a sharp peak is observed. At this potential most of $A$ has been consumed but the speed at which the ratio of $A$ to $B$ is altered at the interface as a consequence of the Nernst equation surpasses the speed at which $B$ can diffuse, resulting in a large concentration gradient at the electrode surface, and, following Fick's first law, in a large flux.

In order to better understand the origins of the two peaks, we analyze the change in the surface concentration of $A$ and in the solution concentration of $B$ over time. Figure 5a shows the dimensionless surface concentrations of $A$ and the ratio of the surface concentrations of $A$ given as:

$$
\operatorname{Ratio~}_{A}=\frac{C_{A}^{0}}{C_{A}^{0}+C_{B}^{0}}=\frac{1}{e^{-\theta}+1}
$$

which are plotted against the dimensionless potential, $\theta$. The ratio is defined in such a way that its values vary between one and zero for simplicity and we see changes in its value relative to the total surface concentration of $A$ and $B$, i.e. $C_{A}^{0}+C_{B}^{0}$, as the reaction progresses. The blue solid line in Figure 5 a represents the surface concentration of $A$ when a linear wave is applied and the green solid line represents the surface concentration of $A$ when a semi-circular potential wave is applied. The light blue and light green dotted lines show the ratio of the surface concentrations of $A$ to the surface concentrations of $A+B$ using the linear and semi-circular waves, respectively. Figure 5b shows the surface concentrations and the surface concentration ratios of $A$ plotted against dimensionless time, $\tau$, using the same color scheme as in Figure 5a. Please note that for direct comparison of Figure 5a and 5b, the time axis in 5b increases to the left.

Looking at Figure 5a, we immediately notice a shift between the plot of the ratios (dotted line) and 


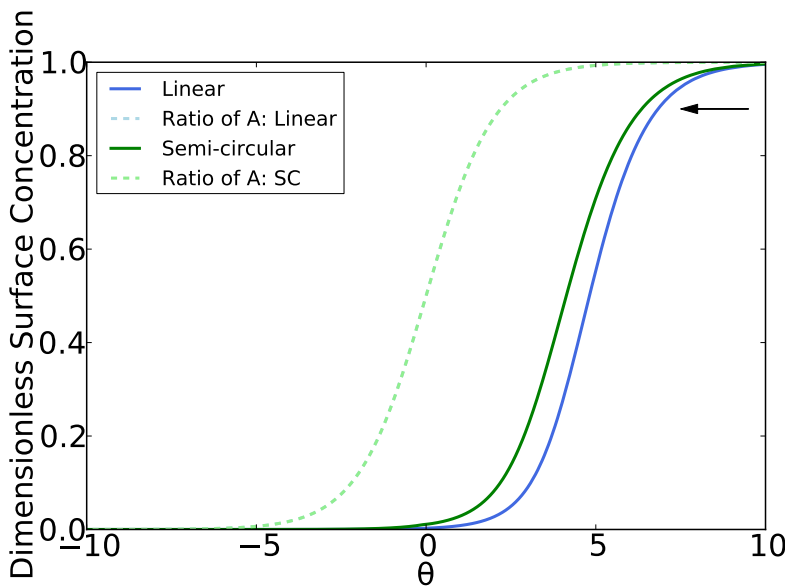

(a) Surface concentration of $A$ and ratios of $A$ (18) plotted against the dimensionless potential, $\theta$.

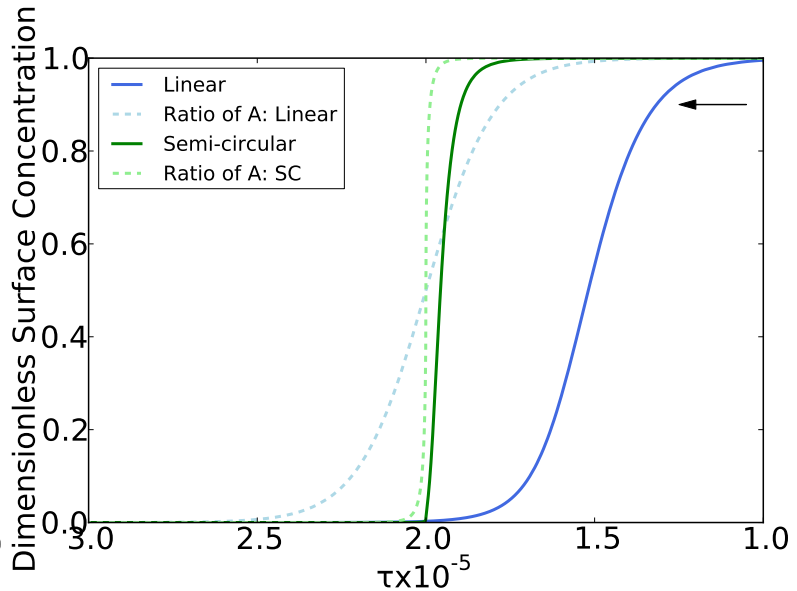

(b) Surface concentration of $A$ and ratios of $A(18)$ plotted against the dimensionless time, $\tau$.

Figure 5: (a) shows the change in dimensionless surface concentration of $A$ and the ratio of $A$ (18) with respect to potential. The blue solid line represents the surface concentration of $A$ when a linear wave is applied and the green solid line represents the surface concentration of $A$ when a semi-circular potential wave is applied. The light blue and light green dotted lines show the ratio of the surface concentration of $A$ to the surface concentration of $A+B$ for the linear and semi-circular method, respectively, which perfectly overlap in (a). The arrow represents the direction of the sweep. (b) shows the change in dimensionless surface concentration of $A$ and the ratio of $A$ with respect to time. Note that for direct comparability, the time axis points to the left. The same color schemes are employed as in (a).

the surface concentrations (solid line) while the two plots of the concentration ratios for the linear and semi-circular potential overlap perfectly. The shift between the ratios and the surface concentration plots can be understood from the diffusion of $B$. At $\theta=10$, we see that a small amount of $A$ is already consumed and $B$ is produced, which diffuses away. Meanwhile the ratio (18) is defined by the Nernst equation throughout the sweep and must hold true at all times. Therefore, more $A$ is consumed as more $B$ diffuses away from the electrode to satisfy the Nernst equilibrium and changes in the surface concentrations are shifted with respect to changes in the ratios. The two ratio plots obtained from the linear and semi-circular potential waves overlap perfectly because, as stated and evident from Equation (18), the ratio is determined by the Nernst equilibrium which is only dependent on the potential and thus the potential wave shape does not affect the shape of the concentration ratio curve when plotted against $\theta$.

When the concentration ratios are plotted against the dimensionless time, $\tau$, in Figure 5b, a significant difference is observed in the curve obtained from the two methods. The linear wave results exhibit the same trend as in Figure 5a whereas the semi-circular wave results show a much steeper curve for both, the surface concentration and the ratio. We further notice that the reaction for a semi-circular potential wave begins at a much later time compared to the linear method and the reaction occurs within a more narrow time frame giving the surface concentration a steep slope.

The observations can be explained by the scan rate difference between the two methods. From Figure 1 , we see that the temporal scan rate of the semi-circular method at the potential at which the reaction starts is already much faster than that of the linear method. As the reaction starts with a faster scan rate, there is less time for $B$ to diffuse, causing a build-up of $B$ at the electrode surface. The build-up of $B$ hinders the reaction which must satisfy the Nernst equilibrium and thus less $A$ is converted until $B$ diffuses into the solution. Comparing the linear and the semi-circular wave, this results in a smaller shift of the latter surface concentration curve with respect to the corresponding ratio. 
We next look at the change in solution concentrations of $B$ over time and plot the solution concentrations of $B$ with respect to dimensionless potential, $\theta$, and dimensionless space, $X$, where figures 6 a and $6 \mathrm{~b}$ correspond to linear and semi-circular results, respectively. The linear method shows one peak extending far into the spatial grid whereas the semi-circular method shows two peaks closer towards the electrode surface. The recorded maximum concentration values differ between the two methods giving the semi-circular method a higher concentration value. The peak in Figure 6a corresponds to the peak current observed in Figure 4. Figure 6b displays two occasions where a build up of concentration occurs: one corresponding to the broad current peak and the other to the sharp current peak. The higher concentration value of $B$ in the semi-circular plot is due to the fact that in the said method, the reaction progresses within a very narrow time frame causing a rapid build-up of $B$ at the interface.

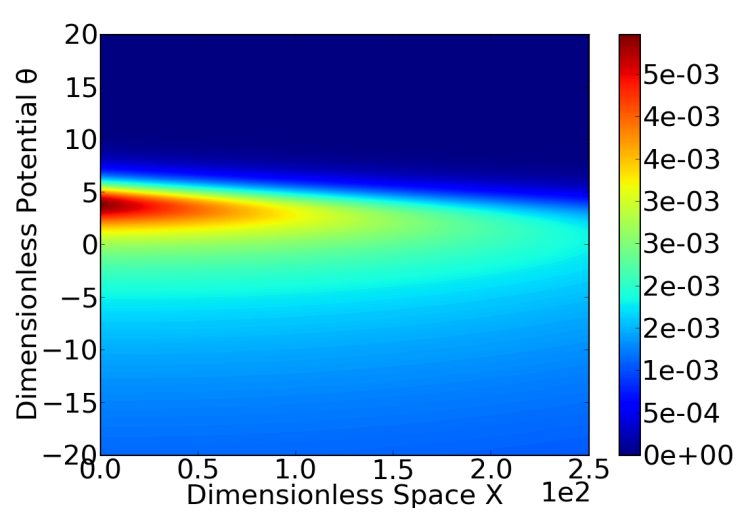

(a) Solution concentration of $B$ with linear potential wave.

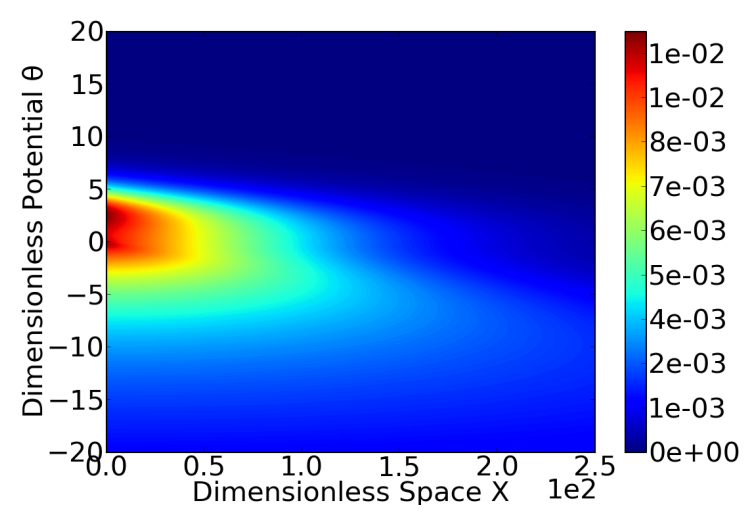

(b) Solution concentration of $B$ with semi-circular potential wave.

Figure 6: The two figures show the solution concentration when (a) a linear potential wave is applied, and (b) when a semi-circular potential wave is applied. The colorbar on the right hand side of the figure represents the dimensionless concentration

To summarize, the double peaks of the semi-circular method in Figure 4 arise from the interplay of different temporal scan rates and the mass transport of $B$ throughout the sweep. The broad peak is due to the increasing scan rate of the potential wave, which consumes $A$ at an increasing rate giving a higher current response compared to the linear method. Between $\theta=3$ and $\theta=1$, the current drops since the low surface concentration of $A$ dominates the effect of increasing scan rate. At $\theta=0$, the scan rate approaches infinity (a jump in the potential value) and thus despite the low concentration of $A$ at the interface, the Nernst equilibrium pushes the reaction to proceed forward very quickly. As a result, a large gradient is produced at the electrode surface and gives the sharp peak.

\subsection{Experimental applications}

In this section we discuss the experimental application of the above method. The biggest advantage of the semi-circular potential wave is its ability to significantly amplify the current as evident from Figure 4. By integrating the voltammogram obtained, one can very easily determine the initial surface concentration of $A$ via:

$$
c_{A}^{s}=\frac{\int_{0}^{t_{\max }} I(t) d t}{F A}
$$


where $t_{\max }$ is the final time, $F$ is the Faraday constant, and $A$ is the area of the electrode.

Figure 2 has further shown that each potential shift produces a characteristic voltammogram that is specific to the formal potential of the analyte. This feature can be exploited when conducting an experimental analysis; Following a careful calibration of an experimental set-up for a specific species, characteristic changes in the shape of the voltammogram can be used to identify interferences with other analytes (assuming only one analyte is present) in a sensing context and may enable the determination of the identity of the species detected. To this end, a sequence of experiments is conducted using solutions that contain known concentrations of known species. The peak currents and voltammetric shapes are determined and then used as a reference in the analysis of unknown solutions.

\section{Conclusions}

We introduce a novel method to run CSV using a semi-circular potential wave, which gives a much more sensitive current response compared the conventional linear wave and hence could be used to detect materials at a lower concentration. The increased peak current makes the analysis of the initial surface concentration easier while the more characteristic voltammetric curves obtained allows the identification of the species under investigation via its formal potential.

\section{References}

[1] J. Barón-Jaimez, M. R. Joya, and J. Barba-Ortega. Anodic stripping voltammetry - ASV for determination of heavy metals. Journal of Physics: Conference Series, 466:012023, 2013.

[2] R. G. Compton and C. E. Banks. Understanding Voltammetry. Imperial College Press, 2010.

[3] K. Wikiel and Z. Kublik. Resolution of the indium peak from the peaks of lead, cadmium and thallium in stripping voltammetry at mercury film electrodes. Journal of Electroanalytical Chemistry and Interfacial Electrochemistry, 165(1):71 - 85, 1984.

[4] J. Davis, D. H. Vaughan, D. Stirling, L. Nei, and R. G. Compton. Cathodic stripping voltammetry of nickel: sonoelectrochemical exploitation of the ni(iii)/ni(ii) couple. Talanta, 57(6):1045 - 1051, 2002 .

[5] W. Yue, A. Bange, B. L. Riehl, B. D. Riehl, J. M. Johnson, I. Papautsky, and W. R. Heineman. Manganese detection with a metal catalyst free carbon nanotube electrode: Anodic versus cathodic stripping voltammetry. Electroanalysis, 24(10):1909-1914, 2012.

[6] W. T. De Vries and E. Van Dalen. Theory of anodic stripping voltammetry with a plane, thin mercury-film electrode. Journal of Electroanalytical Chemistry (1959), 8(5):366 - 377, 1964.

[7] J. Schiewe, K. B. Oldham, J. C. Myland, A. M. Bond, V. A. Vicente-Beckett, and S. Fletcher. Linear-scan anodic stripping voltammetry with thin-film electrodes: theory of the stripping stage and experimental tests. Analytical Chemistry, 69(14):2673-2681, 1997.

[8] J. Buffle. Calculation of the surface concentration of the oxidized metal during the stripping step in the anodic stripping techniques and its influence on speciation measurements in natural waters. Journal of Electroanalytical Chemistry and Interfacial Electrochemistry, 125(2):273 - 294, 1981. 
[9] S. M. Roda, R. D. Greenland, R. L. Bornschein, and P. B. Hammond. Anodic stripping voltammetry procedure modified for improved accuracy of blood lead analysis. Clinical Chemistry, 34:563-7, 04 1988.

[10] P. L. Brezonik, P. A. Brauner, and W. Stumm. Trace metal analysis by anodic stripping voltammetry: effect of sorption by natural and model organic compounds. Water Research, 10(7):605-612, 1976.

[11] Z. Stojek and Z. Kublik. Resolution of anodic stripping voltammetry with thin-film mercury electrodes. Journal of Electroanalytical Chemistry and Interfacial Electrochemistry, 105(2):247 - 259, 1979.

[12] C. Kokkinos, I. Raptis, A. Economou, and T. Speliotis. Disposable micro-fabricated electrochemical bismuth sensors for the determination of $\mathrm{Tl}(\mathrm{I})$ by stripping voltammetry. Procedia Chemistry, 1(1):1039 - 1042, 2009 .

[13] T.M. Florence. Cathodic stripping voltammetry: Part I. determination of organic sulfur compounds, flavins and porphyrins at the sub-micromolar level. Journal of Electroanalytical Chemistry and Interfacial Electrochemistry, 97(2):219 - 236, 1979.

[14] R. G. Compton, E. Laborda, and K. R. Ward. Understanding Voltammetry: Simulation of Electrode Processes. Imperial College Press, 2013.

[15] J. Crank. The Mathematics of Diffusion. Oxford University Press, 1979.

[16] Y. Uchida, E. Kätelhön, and R. G. Compton. Linear sweep voltammetry with non-triangular waveforms: New opportunities in electroanalysis. Journal of Electroanalytical Chemistry, 818:140 - 148, 2018.

[17] E. Kätelhön and R. G. Compton. Testing and validating electroanalytical simulations. The Analyst, 140:2592-2598, 2015.

[18] D. J. Gavaghan. An exponentially expanding mesh ideally suited to the fast and efficient simulation of diffusion processes at microdisc electrodes. 2. Application to chronoamperometry. Journal of Electroanalytical Chemistry - J Electroanal Chem, 456:13-23, 091998.

[19] W.T. De Vries and E. van Dalen. Linear potential-sweep voltammetry at a plane mercury- film electrode. Journal of Electroanalytical Chemistry and Interfacial Electrochemistry, 14(3):315 - 327, 1967.

[20] C. M. A. Brett and A. M. C. F. O. Brett. Dc anodic stripping voltammetry at mercury thin film electrodes: the shape and position of the stripping peaks at hydrodynamic electrodes. Journal of Electroanalytical Chemistry and Interfacial Electrochemistry, 262(1):83 - 95, 1989.

[21] J. C. Ball and R. G. Compton. Anodic stripping voltammetry at hydrodynamic mercury thin film electrodes. Numerical simulation of stripping peaks for reversible processes at uniformly accessible electrodes. Electroanalysis, 9(10):765-769, 1997. 